Healthy foods prepared at home: Diet and support as protective strategies during pregnancy for Hispanic women

Allison L. Hopkins ${ }^{1 *}$, Michelle Yeoman ${ }^{2}$, and Cheryl Ritenbaugh ${ }^{3}$

${ }^{1}$ Department of Anthropology, Texas A\&M University, College Station, Texas, 77843 USA

${ }^{2}$ Departments of Veterinary Integrative Biosciences and Anthropology, Texas A\&M University, College Station, Texas, 77843 USA

${ }^{3}$ Department of Family and Community Medicine, University of Arizona, Tucson, Arizona, 85721 USA

*Allison L. Hopkins, 4352 TAMU, College Station, TX 77843-4352, hopkins@tamu.edu 
Abstract and Keywords

Birth outcomes tend to be better among Hispanics than among other ethnic groups, even when matched for poverty and education, and foreign-born Latinas compared to their U.S. born counterparts. These patterns suggest that sociocultural factors exhibited by recent immigrants have the potential to protect birth outcomes against the instability of minority and low socioeconomic status. To discover potential sociocultural factors, a pilot qualitative study was carried out in Tucson, Arizona with 18 Hispanic mothers. The two most prevalent factors reported were 1) a healthy diet prepared at home from minimally processed ingredients, and 2) constant and comprehensive social support. When comparing responses related to diet by interview language preference, a proxy for acculturation, there was very little difference between participants who interviewed in Spanish and those who interviewed in English. This result may be explained by greater maternal social support and higher education levels among those who interviewed in English.

Hispanic Health Paradox, pregnancy, diet, social support, immigration 
Introduction

Although infant mortality rates in the United States have decreased (CDC), not every group has benefitted equally from this trend. Some regions of the United States have infant mortality rates as high as developing or conflict-strife countries. For example, one ZIP code in Indiana has an infant mortality rate of 24.1 among African Americans (Bruce 2017; ISDH), which is higher than Columbia (14.1), Turkey (18.2), and Syria (15.2) (World Bank). Health disparities are differences in the prevalence of disease and in the opportunities to experience ideal health by socially disadvantaged groups of people (CDC 2008). Generally, health disparities correlate highly with measures of access to resources.

However, Hispanics provide an exception to this generality as they tend to have better health and mortality outcomes than non-Hispanics despite higher rates of poverty, less educational attainment, and less access to health care (Arias 2010; Franzini, Ribble, and Keddie 2002). For example, Hispanics have lower overall death rates than non-Hispanic whites for cancer and heart disease-the two leading causes of death in the U.S (Dominguez et al. 2015). Although Hispanics share similar socioeconomic conditions with non-Hispanic blacks, infant mortality rates are lower for Hispanics (5.0) than blacks (11.3), and are similar to rates for non-Hispanic whites (4.9) (Mathews and MacDorman 2011). This paradoxical relationship, coined the “Hispanic Health Paradox” (Markides and Coreil 1986), has been documented extensively, yet causes for this paradox remain unclear. The potential under-reporting of Hispanic deaths as the result of the "salmon bias" and healthy migrant effects, in addition to risk profiles, may contribute to, but does not fully explain the paradox (Palloni and Morenoff 2001; Franzini, Ribble, and Keddie 2001; Ceballos 2011). 
Sociocultural factors, such as family structure, lifestyle behaviors, and social support, provide promising plausible explanations because these factors have the potential to provide a protective barrier against poverty and poor socioeconomic conditions (Franzini, Ribble, and Keddie 2001; Arias 2010). Further support of the importance of sociocultural factors comes from migration studies, which exhibit a general pattern that foreign-born Hispanics have better health outcomes upon first arriving in the U.S. as compared to their U.S. born counterparts; however, with increasing duration in the U.S., their health status declines to approximate Hispanics born in the U.S (LaVeist 2005; Singh and Yu 2002; Lara et al. 2005). This negative correlation between health and length of time residing in the United States is particularly consistent among birth outcomes, including birth weight and term length, and in substance abuse and dietary behaviors (Lara et al. 2005; Hummer et al. 2007; LaVeist 2005), both of which are known to impact birth outcomes (Abu-Saad and Fraser 2010; Bloomfield 2011; Behnke et al. 2013; Cobas et al. 1996). Although this trend is consistently observed in different health measures, Hispanics are a heterogeneous group and the health-related behavior changes that occur post-migration are complex and still not clearly understood (Lara et al. 2005).

One of the major issues that contributes to the difficulties in determining health-related behavior patterns experienced post-migration is that acculturation, generally defined as the degree to which an immigrant individual or group adheres to the sociocultural beliefs and values of the host group, is a complex multidimensional process. According to Berry's four-model theory (1997), acculturation is divided into four typologies: marginalization (isolation from both natal and host cultures), separation (adherence to natal and separation from host), assimilation (adoption of host and departure from natal), and adaptation (adoption of host and adherence to natal). Of course, acculturation is not a unilinear construct—individuals may practice difference 
acculturation typologies depending on the social context, and these practices may change with time.

Because acculturation consists of multiple sociocultural dimensions, measuring acculturation can often be problematic. Multidimensional scales that enable the capturing of all the dimensions can often be burdensome to implement. Thus, many studies use language or length of residence as practical and efficient proxies for acculturation (Thomson and Hoffman-Goetz 2009). Some researchers contend that unidimensional scales fail to capture the nuance and complexity of acculturation (Lopez-Class, Castro, and Ramirez 2011; Unger et al. 2007). However, others assert that language is a robust measure of acculturation, particularly in relation to health attitudes and behaviors (Deyo et al. 1985; Shin and Maupome 2017; Arcia et al. 2001). In a principal component analysis with 150 Latino families, Arcia and colleagues (2001) found that language preference most strongly correlated with levels of acculturation. Similarly, Shin and Maupome (2017) found that Spanish language preference strongly correlated with less acculturation as measured by a multidimensional psychological and behavioral scale.

Another issue that contributes to the complexity in understanding health-related behavior change post-migration is the role of social support. The beneficial role of social support is well supported and associated with better health outcomes (Berkman 2000; Sarason et al. 2006). In general, social support can be classified into three types: 1) emotional, informational, and instrumental (Collins et al. 1993). Some researchers have debated whether the protective effects of social support align with a main-effect model, in which social support and networks provides an overall positive effect by promoting an individual's sense of well-being and self-worth. Alternatively, others posit that social support most closely aligns with a buffering model, in 
which social supports acts to moderate and buffer the negative consequences of stress. In reality, both models are likely accurate (Cohen and Wills 1985), depending on the context.

Social support is also correlated with better birth outcomes, including higher Apgar scores (Collins et al. 1993), higher weight infants at birth (Collins et al. 1993; Feldman et al. 2000), easier labor and delivery (Collins et al. 1993), and lower maternal depression (Gress-Smith et al. 2013). Higher social support also appears to moderate the negative effects of long residency in the US and worsening diet (Harley and Eskenazi 2006). Importantly, higher familial support is consistently associated with Latino cultural identity (Almeida et al. 2009; Rodriguez et al. 2007), but seems to diminish with longer residence in the US and acculturation (Almeida et al. 2009).

One area of research investigates the role of individual relationships versus social networks and cohesion. Feldman and colleagues (2000) found that multiple types of support predicted higher birth-weight infants. By contrast, Mulvaney-Day, Alegria, and Scribney (2007) found that neighborhood social networks did not improve maternal physical and mental health—instead, individual and personal social connections appear more important. Fleuriet (2009) makes a compelling argument that one type of categorical support is not better than another, but instead depends on the needs and desires of the pregnant woman. Social support may also moderate the negative effects of increased length of U.S. residence on diet quality among pregnant women of Mexican descent (Harley and Eskenazi 2006).

The goal of this study was to carry out a qualitative exploration of the complexities of healthrelated behaviors as they relate to migration with special attention to sociocultural factors that may be influencing birth outcomes from the perspective of Hispanic mothers in Tucson, Arizona. 
Methods

Under a protocol approved first by the University of Arizona IRB (12-0708) and later by Texas A\&M IRB (IRB2016-3009D), Hispanic mothers were recruited from the first author’s (ALH) professional network and the waiting room of the Family Community Medicine Mobile Health Clinic in Tucson, Arizona. Recruitment efforts centered on maximizing demographic variability, particularly language preference and country of origin, and continued until the point of saturation of information provided by the participants. A total of 18 women were recruited for participation. The original goal was to interview Mexican origin mothers in order to tap the breadth of their ideas about the Hispanic Health Paradox, and possibly reach saturation on some constructs. However, saturation was reached after only a few interviews, so additional interviews with mothers from other countries and lengths of stay in the U.S. were sought to determine what might be sources of variation in the constructs already reported.

Interviews took place between October 2012 and November 2013 at locations convenient to participants, including their homes, places of employment, or study rooms at local libraries in Tucson, Arizona. Each recorded interview lasted between one and one-and-a-half hours, and half were conducted in English and half in Spanish based on the respondent's preference. After the consenting process, the first author (ALH), who conducted all the interviews, described the Hispanic Health Paradox in relation to pregnancy and birth outcomes. Respondents were then asked why they thought women who recently emigrated from Latin America tended to have healthier babies than women who have lived longer in the United States. After the participants were given ample time to respond to the open-ended question, they were probed with questions focusing on health behaviors that have been associated with pregnancy outcomes and 
immigration in the scientific literature or that other previously interviewed participants had mentioned. At the end of the interview, the participants responded to a one-page demographics survey. The results of that survey were summarized using descriptive statistics.

Interviews were transcribed and then analyzed using ATLAS.ti v8 software to identify trends and factors that respondents attributed to the epidemiological pattern of greater maternal and fetal health among new immigrants. Data were analyzed using the noticing, collecting, and thinking (NCT) model of computer-assisted data analysis, which consists of two levels of analysis: descriptive level and conceptual level (Friese 2014, 12-22). In the descriptive-level of analysis, a hierarchical coding approach was utilized, which included defining lower-level descriptive codes followed by the creation of higher-level conceptual codes. In order to ensure a robust code system and intercoder reliability, ALH and MY each coded the same interview. They then discussed discrepancies, came to an agreement on how the discrepancies should be coded, created higher-level codes, and generated operational definitions for all codes (Friese 2014, 133135, 140-156). After reaching consensus, ALH and MY coded two more interviews independently, then repeated the above process, modifying and refining codes and parameters as needed. The result of this process was convergent validity between both coders.

In the conceptual-level of analysis, research questions are answered by using a variety of analytical tools on the coded data (Friese 2014, 18). This level of analysis started with a review of the results of a basic query of key codes, including "Food: healthy," "Food: processed," “Food: homecooked,” “Food: honor cravings,” “Food: time/convenience,” “SS: food preparation,” and "SS: general social support." Illustrative quotes were identified and translated into English by ALH when necessary. Next, separate reports were created based on interview language, which was used as a proxy for length of time residing in the U.S., and queried using 
the key codes previously mentioned. The results were reviewed and differences between language groups were noted. Lastly, to determine possible code associations, a co-occurrence table was generated and analyzed to identify codes that frequently occurred together. Cooccurrence is defined by spatial context, such as when codes occur within the same quotation or nearby quotations (immediately preceding or following).

Results

Study Participants by Interview Language

The sample consisted of 18 self-identified Hispanic mothers residing in Tucson, Arizona (Table 1). In order to gain a better understanding of differences between participants with different language preferences, the sample was divided by the 9 participants who chose to interview in English and the 9 participants who chose to interview in Spanish. The age ranges and averages were similar for those interviewed in Spanish (27-75 and 43 years) compared to those interviewed in English (31-74 and 47 years) (Table 1). The Spanish language interviewees’ educational attainment was divided between the low end of the spectrum (11\% no formal education and 33\% middle school) and the high end (56\% postsecondary education). The educational attainment of English language participants was concentrated at the high end with $11 \%$ receiving a high school diploma and the remaining $89 \%$ participating in postsecondary education. More of the Spanish language participants were from Mexico (67\%) than the English language participants (22\%), and more of the English language participants were from the United States (33\%) than the Spanish language interviewees (0\%). There was fairly equal representation 
from the rest of Latin America in both groups, although the specific country of origins varied. Among the $1^{\text {st }}$ generation participants, the average number of years residing in the United States was double for those who interviewed in English (30 years) as compared to Spanish (15 years), although the range was very similar (2-50 and 2-46 years, respectively). An equal number of participants (89\%) were raised in cities with 30,000 or greater people in both groups. Those who interviewed in Spanish mostly preferred to speak it in the home (89\%), whereas those who interviewed in English were more diverse in their home language preferences with the majority having no preference between the two languages (67\%) and of the remaining slightly more preferred English (22\%) to Spanish (11\%).

\section{Overall Observations}

A healthy diet was the most important factor reported to impact birth outcomes. For example, Linda emphasized the importance of preparing healthy foods at home:

“Well, I think the home cooking, you know they don’t buy too many carry outs or pizza or food. I think we eat healthy, Mexican food like chicken soup, tortilla soup, just adobado [seasoned], tasty, but it's not saturated with fats ... I think that is the main thing, we eat a lot of veggies and fruits.”

Like Linda, most participants (72\%) reported that a healthy diet—consisting of plentiful fruits and vegetables with limited meat—was essential for maternal and fetal heath. For $44 \%$ of respondents, healthy diet was their primary response, although one respondent was unsure what 
led to a healthy pregnancy. Many participants reported that Latin American cultures, as opposed to American culture, emphasize cooking healthy meals at home. Co-occurrence data analysis revealed that healthy foods were frequently associated with Latin American culture (occurring together 21 times), and home cooking (18) (Figure 1). Processed food was more frequently associated with American culture (7) than Latin American culture (3).

The remaining 50\% reported that social support was the primary factor, with $61 \%$ spontaneously reporting it as the primary or secondary factor. Intriguingly, the initial emphasis on diet or social support correlated with interview language preference. Fifty-six percent of participants who interviewed in Spanish provided diet as their initial response, while 33\% reported social support first. In contrast, 33\% of those who interviewed in English provided diet as their initial response, with $67 \%$ providing social support.

Co-occurrence analysis revealed that general social support was associated with mothers (29), family in general (15), Latin American culture (15), other female relatives (10), and husbands (10) (Figure 1). Husbands (10) were also associated with a lack of support, as were the biomedical healthcare system (7) and American culture (5).

The remainder of the results section delves further into factors that participants stated impact birth outcomes, along with similarities and differences in responses between English and Spanish language preference.

\section{Healthy foods prepared at home}

Members of both language groups emphasized that eating healthy was an essential part of a healthy pregnancy. Some reported following the same diet as they did while not pregnant, while 
others adopted a healthier one after becoming pregnant. Rosy explained in English that she battled with morning sickness early in her pregnancy, but then adopted a healthy diet later: "After that phase of being disgusted with everything, I ate pretty healthy: pasta, fruits and vegetables.”

The participants in both groups had very similar ideas regarding essential ingredients for a healthy diet during pregnancy. These ingredients included a variety of protein sources like beans, eggs, chicken, and beef; some carbohydrates like rice and tortillas; and plentiful fruits and vegetables. Amira referred in Spanish to a list of basic ingredients that were essential to a healthy diet: "Well the basics, that is not a lot of meat—because well, we couldn't, but milk, eggs, beans, a lot of soup, tortillas ... vegetables, fruits also a lot.”

Although participants in both groups shared similar ideas regarding a healthy diet, a few differences are apparent. English language participants described a wider variety of vegetables and fruits than Spanish language participants. Those in the Spanish group reported consuming more breakfast cereal. A few Spanish language respondents referred to drinking tea, particularly chamomile; however, no one in the English group mentioned tea. Sandwiches and leafy greens based salads were referenced much more frequently by English language participants than Spanish. A few participants in the English group characterized Latin Americans as eating less bread, pastries, and desserts than Americans, although the Spanish group did not make that distinction.

Some participants, like Alexandra did in English, emphasized that a healthy diet consisted of fresh foods without added chemicals: "We eat more like natural food, like everything with no chemical, maybe that is what it is too. We eat a lot of fresh stuff.” Julia also emphasized in English the importance of fresh foods when she described Mexican cultural practices around 
food: “In Mexico by nature they are very fresh people. If it’s not the maid that's making it, it’s the mom that's making it. They go to the market, and they get the things fresh, and there's not a lot of frozen precooked preservative stuff going on.” Both groups preferred fresh ingredients; however, respondents did consume a limited number of canned, frozen, and dried items.

Participants also listed foods to avoid. Both groups found pre-packaged meals to be extremely unhealthy and recommended limiting fat, salt, and sugar consumption. In particular, processed foods were seen as a potential mechanism by which food could negatively impact health of the population in general. Bea expressed in Spanish concerns that toxins and chemicals in foods could cause long-term health problems:

So all this about food is very important. If it is altered, chemicals with transgenics and I don't know what, your body is going to react. It's like bombs that you put in. So, now I know, and there are a lot of studies, that through the problem of chemicals and all [that] has been generated, there are many laboratory illnesses, diabetes and all that.

Rosy also discussed in English the potential harm of toxins on fetal health:

In pregnancy you have to be very careful whatever toxins or other things that you [consume]. If you're not pregnant, if you're an adult and your brain has developed, and your body has developed, is not affect you as much as if you are pregnant, and whatever you put in your body goes through the placenta to the fetus. 
Participants in both groups also discussed food quality by using terms such as locally sourced and organic. For many women, as illustrated by Rosy in English, organic and locally sourced foods were viewed as more nutritious and healthy:

I think especially in pregnancy and for young children, like organic, certain organic products are important like some fruits and vegetables, and you can find the dirty dozen and the clean whatever. I can't remember the name of the organization that puts out [the list]. Celery and strawberries, there are certain things you should have organic versus other things that you are peeling it doesn't really matter. Definitely be careful where the meat is coming from and then the fish, there's all sort of problem with the fish and the mercury and all that, but yeah, it's complicated.

Both groups reported that fresh, locally produced food was more accessible in Latin America than in the U.S. Rosy stated in English that Argentina had better sources of meat and described her difficulties obtaining grass-fed beef for her pregnant daughter when in Arizona:

The sources of the food [in Argentina] I think are much better than the sources of the food that we have now here even today, you know all meat, and I don't know about chicken in Argentina, but beef is pretty much grass feed. Here I tried, my daughter is pregnant, and so I've been trying to get, and she eats meat, so trying to get grass fed beef in Tucson is like an ordeal.

However, in Spanish Elvia noted that this trend is changing in urban parts of Latin America: 
There in Navojoa, a small city almost town, in the outskirts there are many people that cultivate. For example, Sundays in the morning we were going to get the crops and buy fresh carrots, onion, lettuce, potatoes, squash. We get them, but we did not plant them ourselves. We knew that people were harvesting there and it was very normal. Where we lived in Obregon it is no longer like that.

Partly because of accessibility and affordability, participants stated that Latin Americans had healthier and more nutritious diets than Americans (Figure 1). Both groups also reported that organic, locally sourced foods are more expensive in the U.S. than in Latin America. As Bea described in Spanish, the decision to consume organic foods comes with increased costs:

The organic, for example, here is more expensive. We opt for organic food ... It would be cheaper to buy all of our food in Food City or in other places. The problem is the question of where the food is from and the problem of the chemicals and everything, so looking for everything organic also demands a higher budget.

Beans and soups were identified as inexpensive foods by both groups, as well as foods available on family ranches and farms in Latin America. Participants also implied that no matter how poor their families were in Mexico, they still had the ability to eat healthy foods. Amira recalled in Spanish growing up in Mexico: "There [in Mexico] we do not have so much money . . . but we simply ate well and always had something to eat, the most inexpensive so beans and broth, we did not eat a lot of meat.” Despite their relative poverty, Amira’s family enjoyed 
access to affordable, nutritious foods: "[Our diets were] very healthy, and you eat beans and rice and, if you can, milk, if not, no. My mom was born in Sinaloa on a ranch. My grandparents had cows and everything, so they always had milk, they had eggs, but they were always very poor.”

The participants expressed an awareness of the different economics of processed and whole foods in the U.S. versus Latin America, which can greatly impact consumption patterns. They noted that whole foods tend to be less expensive in Latin America and more expensive in the United States, whereas processed foods are the reverse. Some participants stated that homecooked foods are healthier, tastier, and cheaper. Carmen said in English, "I mean financially it is just easier to not [go out to eat] ... But yeah I just try more to just avoid it because like spending $\$ 7$ on a meal when I can make stuff at home, it’s cheaper.”

Some participants reported that Latin American diets are healthier because they rely on home-cooked meals rather than fast food (Figure 1). Julia said in English, "When I was pregnant and going through, I cooked, I made my own food from scratch ... I think it is a big part of the culture, too, is that they do cook a lot from scratch.” Soup, in particular, was often referenced as a healthy food that was cooked at home: "Everything was made from scratch ... We always have vegetables, we always have our soups and salads ... nothing but home cooked food.”

The importance of home cooked foods was also linked to preventing illness, as described by Alexandra in English: “We don’t go to restaurants, my family, so my mom cook every day, three times a day, so no restaurant food. I think that's why we don’t get that much sick neither.” 
In addition, Spanish language participants emphasized that home-cooked foods can prevent complications during pregnancy. Elisa said in Spanish, "I tried to cook at home ... I believe that it served me and I did not have any serious or difficult complications during the pregnancy.”

Some participants dined out occasionally, no more than once or twice a week, and usually as part of a celebration or because of a child's preference. Alexandra, a participant interviewed in English, prefers to dine at home, but sometimes acquiesces to her child's wishes: "The small one, she likes to eat out. I don't know why cus we raised them eating at home, but I think is maybe friends, they follow their friends and they always wanna go out to eat.” Amira also prefers to dine at home. "I prefer to eat at home than to go eat somewhere. Only if we really want to or we are going to celebrate something, so then we go, but I prefer to make the food at home,” she said in Spanish.

The English language group also referred more frequently to strategies for dealing with the issue of convenience. By preparing and freezing large quantities of food or eating leftovers, they were able to make fresh, home cooked food more convenient. Julia said in English, "I would cook in bulk and then freeze it so that I would have the convenience of just grabbing and zapping it in the microwave.” Carmen shared in English that leftovers were a convenient way to serve home-cooked meals without the burden of preparing a new meal: "He'll [her husband] make a steak, and I'll eat half of it for dinner and then leave the other half for the next day and eat that with more of the zucchini, so just some sort of repeat of whatever we had at night.” Interestingly, no one in the Spanish group mentioned using these time-saving strategies. 
Celia also stated in English that self-care was emphasized more among recent immigrants, which affected women's diets and nutrition:

I believe it has to do with acculturation, probably cus they're not as acculturated, so they're not accustom to the Western, or maybe a better term Americanized, way where it is all about fast food. Women, I don't know, not that we don't take care of ourselves more here in the United States, but I just think it is our way of living. Whereas, you know, if you're living in Latin America, I think there is more of a tendency to ... just eating healthier where you're making stuff from scratch at home and not doing the fast food thing.

Although participants emphasized the importance of following a healthy diet cooked at home, many also experienced difficulties maintaining the ideal diet. Accessibility, affordability, and time management all affected women's abilities to follow the perceived ideal pregnancy diet. As shown below, extensive social support often enabled women to enjoy healthy meals cooked at home.

\section{Constant and Comprehensive Social Support}

For Rosy, becoming pregnant with her second child while away from her support network proved to be a trying experience. "I was in the middle of residency training, and I got pregnant with my son,” she said in English. “We had moved away from my hometown so I didn’t have family ... Even though I was a physician and my [then] husband was also a physician ... I still 
had most of the, you know, caring for the baby and the household was mine ... my [then] husband was a little useless at that point.”

Alexandra also shared in English that she received social support with her first child, but then moved away from her parents and extended family:

"With my first baby, my experience it was kind of nice cus I have all my family around me. Everything I needed, it was there. When I move here, I don't have nobody. Is a lot of stuff that I didn’t do with my second baby that I did with my first baby in my country. So could be family, I would say family around me, family and friends, and here was like nobody just my husband.”

Social support was perceived by both groups to be a main influence on pregnancy outcomes. Participants described social support that was often constant and comprehensive, including cooking and childcare. Although a few Spanish-speaking participants participated in Women, Infants, Children (WIC) while pregnant and the majority of participants mentioned prenatal visits with a doctor, almost all the food-related advice and support they received was reportedly provided by family and friends. Victoria confided in English that she received emotional support from her mother and aunt, which she found invaluable as a first-time mother: "In the beginning, just like the first month my aunt and my mom [helped me]. Just kind of guiding you through the first couple of days and make sure you're on track. It's nerve racking having your first kid and not knowing.” 
Many participants (67\%) reported that their mothers were their primary support person, especially during the birthing and postpartum period (Figure 1). Julia shared in English that Mexican culture emphasizes the role and importance of mothers: “My mom is my mom, I don’t know what I would do without her. In that culture we're always just like, that's your main caregiver, if they are willing and able, meaning healthy, their disposition is good, and you have a good relationship with them, that is the first person you take your kids to be cared for.”

If the participant's mother lived in the same town or the pregnancy was difficult, the mother's support may begin during the pregnancy period, such as the case with Linda who said in English:

After they diagnosed me and they say they were going to sew my uterus up so the baby wouldn't drop, and I had to be in bed rest and they gave me all kinds of hormones, like I said it was like a research, nobody knew what was going on. Then we asked my mom if she could come and be with us. She was retired, and she wasn't working anymore so she came, she said yes. We sent her the ticket, and she came and stayed with me the seven months.

Linda continued to receive social support from her mother with her other pregnancies, from cooking and cleaning to emotional support:

My mother used to come and take care of me seven months out of the year out of my pregnancies ... She did everything. She cooked, she cleaned the house—not every little 
corner but she straighten up the house because she was older already. But she cooked, and she did the dishes at night, and she kept me company.

At the time of the interview, Carolina's mother had just arrived from Mexico to provide social support: "She lives in Mexico and this week, two or three days ago, she arrived from Mexico to help me. To be with me during labor to help me, to be present there helping me be strong, to help me if I want to walk.” Two-thirds of participants received social support in the form of food preparation. Carolina expressed gratitude in Spanish for the help her mother provided, which included childcare and cooking:

She helps me here in the house, also with the children because I have two other children and she helps me. For example, she does not let me cook the first 40 days [after labor]. She tells me that I should not cook. I should not get close to the hot stove and have it hitting me in the belly ... She is a big help, truly.

The individuals who provided support varied from mothers to husbands and other family members (Figure 1). Melanie referred to her husband when she said in Spanish, "Between the two of us we cleaned. Between the two of us we made food. Yes, he helped me a lot [while she was pregnant]. He helped me take care of the children. He helped me a lot.” Carmen shared in English that her support network was fairly large and included extended family: "My dad is not that supportive, but I have a super uber supportive mom and grandmother and uncles and aunts.” 
Many participants, like Carmen, received social support from extended family. Aimee referred to social support in Panama when she said in English, "Always the extended family was helping out with the other kids, with cooking, and allowing the mother to do the things she needed to do and if she didn't have time other people would help out.”

Some participants, like Julia, a second generation Hispanic who was interviewed in English, believed that Latin American cultures tend to be more cooperative and less stressful (Figure 1):

The culture in general is a very tight culture, so you know how stress is a very big part of our life, and I think that we're not as alone as our counterparts here. A lot of my friends that are Americans, they're very on their own, it's like them and their husband, and that's it and if their husband is no good, then is just them against the world.

However, when comparing responses related to social support, some interesting differences were revealed between the English and Spanish language groups. More English language participants reported having general support than did the Spanish. Seventy-eight percent of English language participants reported having constant support, especially from mothers, other female relatives, and friends. Spanish language participants relied more heavily on husbands and girlfriends for general social support, with 44\% reporting so. Only 1 English language participant did not refer to social support. Additionally, one-third of the Spanish language participants made no mention of social support but instead focused on diet.

Differences in social support may be related to whether or not the respondent worked outside the home during pregnancy, in which case support may be more explicitly needed. Over half 
(56\%) of the English group worked outside the home during pregnancy, whereas only 22\% of the Spanish language participants did. Of the two Spanish language respondents who worked outside the home, at least one did so because of financial necessity. Most of the English language participants who worked outside the home stated that, as professionals, they did not want to take an extended break from their careers.

\section{Discussion}

\section{Healthy Foods Prepared at Home}

The findings suggest that a healthy diet consisting of fresh, minimally processed foods prepared at home is a strategy used by the study participants for reducing the risk of delivering a baby with poor birth outcomes. There were some minor differences when comparing the detailed information provided on diet between those who interviewed in English and Spanish, but overall they reported very similar eating habits during pregnancy. Both groups emphasized the importance of eating a variety of protein sources like beans, eggs, chicken, and beef; some carbohydrates like rice, tortillas or bread; plentiful fruits and vegetables; and a variety of soups. Additionally, both groups highlighted the importance of limiting salt, sugar and fat intake. The consistency in reported eating habits by both groups suggests its level of importance-especially when combined with the major challenges to accessibility in the U.S. of the type of healthy food described as reported by both English and Spanish language groups. Additionally, several English language participants developed strategies for making home cooked, fresh foods more convenient by preparing and freezing large batches for easy consumption at a later time or consuming leftovers. These practical, time-saving strategies could be the foundation for an intervention on healthy eating among pregnant women. 
Reviews of the epidemiological literature support the participants' identification of the importance of a healthy diet on birth outcomes. The research demonstrates that adequate maternal nutrition and consumption of specific micronutrients, particularly folic acid, are important for fetal development and birth outcomes (Abu-Saad et al. 2010, Bloomfield 2011, Wu et al. 2012; Blumfield et al. 2012). This is especially true during the periconception period when undernutrition, by not meeting the recommended levels of nutrients based on the dietary reference intakes (Medicine 2006), can have the greatest influence on term length and birth weights, two commonly measured birth outcomes. Some participants in this study specifically mentioned having the same healthy diet prior to and after conception, whereas others clearly indicated a positive change in diet after conceiving.

Less research, however, has been done on the dietary trends of Hispanics residing in the United States for varying lengths of time, especially in relation to pregnancy. There is some evidence that individuals residing in the U.S. for longer periods of time consume less fruits and vegetables, rice and beans, grains and legumes, fish and seafood, tortillas and bread, fiber and vitamins; they also consume more sugar-laden drinks and alcohol, fast food (including hamburgers and pizza), processed foods, meat, fat, and sugar (Pérez-Escamilla and Putnik 2007; Ayala, Baquero, and Klinger 2008; Gray et al. 2005; Chávez et al. 1994; Dixon, Sundquist, and Winkleby 2000; Himmelgreen et al. 2007). A few pregnancy specific studies support this trend of worsening dietary quality with increasing length of time in the United States (Schaffer et al. 1998; Cohen et al. 2001; Harley, Eskenazi, and Block 2005).

However, the qualitative data presented in this study did not support this trend, as English language participants reported consuming a high quality diet very similar to their Spanish language counterparts. A few studies show a similar lack of relationship between pregnancy diet 
and length of residence in the United States for Hispanics (Kieffer et al. 2013; Gutierrez 1999). Kieffer and colleagues (2013) conducted a study of periconception dietary intake among 234 Mexico-born immigrants from 2004 to 2006 in Detroit, Michigan, which showed no association between dietary intake and length of residence in the United States. Similarly, Gutierrez (1999) found no differences in dietary adequacy and pregnancy outcomes of gestational week at delivery and infant birth weight between 36 Mexico-born and 10 United States born pregnant adolescents from the San Francisco Bay Area between 1994 and 1995. The factors attributed to this lack of difference were the persistence of culturally based protective factors, including an increased consumption of traditional dietary staples during pregnancy and a strong family support system to help the mother practice self-care for the sake of the baby during the pregnancy. These factors were also linked to the high value placed on motherhood among Hispanics that endured across the $1^{\text {st }}$ and $2^{\text {nd }}$ generation participants.

In addition to the factors mentioned by Gutierrez (1999), the lack of difference in dietary patterns between the Spanish and English language participants in this study may also be impacted by the mediating role of socioeconomic status and education on the trend of worsening diet with length of time in the U.S., as found in the literature (Fitzgerald 2010). Although socioeconomic status was not captured in this study, educational levels were higher among the English language participants, which may have mitigated the negative impacts of residing in the U.S. for longer than the Spanish language participants.

\section{Constant and Comprehensive Social Support}

Besides a healthy diet, constant and comprehensive support, especially from the pregnant woman's mother, were considered key factors in having a positive pregnancy outcome by many 
of the participants in this study, regardless of language preference, and similar to that described by Gutierrez (1999). Harley and Eskenazi (2006) also observed in their 1999-2000 study of Spanish speaking pregnant women in Salinas Valley, CA that social support protected women against some of the negative pregnancy behaviors, especially poor diet, that have been reported as increasing among women of Hispanic descent who have resided in the U.S. for longer periods of time. The majority of the respondents in this study received social support with cooking healthy meals, especially those who worked outside the home, which may help explain why dietary patterns were so similar between the Spanish and English language participants.

Another explanation for the similarities in diet between participants interviewed in English and Spanish might be the positive association between social support and income and education, which has been identified by Harley and Eskenazi (2006) in the previously described study and Wakeel and colleagues (2014) in their 2007 study of almost 3,000 pregnant Hispanic women. This pattern was also observed in the current study with English-language participants having higher education and more extensive social support as compared to Spanish language participants.

In many cases, the ideal type of pregnancy support described by the current study participants was not achievable, so it was modified in different ways. For example, when a participant's mother did not live nearby, she would come to visit and provide support during critical time periods; this was especially the case among English language respondents. When a respondent's mother was unavailable, other people in the participant's personal network provided support, including other relatives—especially husbands and friends. This was particularly the case among the Spanish language participants, as some reported that their mothers still lived in Latin America and were unable to visit even for a targeted period of time. 
More English language participants worked outside the home during pregnancy, which may explain a greater need for social support. Lower levels of social support reported by Spanish language participants may also be the result of recent establishment in a new area with no or few prior personal ties, as some Spanish language respondents mentioned.

A few previous studies reported similar differences in support between immigrants and their U.S.-born counterparts. Between 2001 and 2003, Viruell-Fuentes and Schulz (2009) compared experiences with social support between 20 first-generation Mexican immigrant women and 20 second-generation Mexican Americans residing in Detroit, Michigan. Their findings suggest that first-generation women relied on a small group of close family for support, whereas secondgeneration women had a much more extensive social network that includes extended family and life-long acquaintances. Viruell-Fuentes and colleagues (2013) also carried out a study in Chicago during 2002 comparing the degree of different types of social ties experienced by 502 foreign-born Latinos as compared to 290 U.S.-born Latinos. The overall finding of their study was that Latinos born in Latin America had significantly lower levels of social ties in comparison to those born in the U.S.

The main limitations of this study are its small size and purposive sample, including the even smaller sample sizes in various SES groups, and the necessarily retrospective nature of the collected data. Further, all data were collected in a single location by a single interviewer, and thus broadening data collection to other communities and more interviewers would be worthwhile. However, the surprising consistency of the participant reports, even across different Latin American countries of origin, can serve as a starting point for more systematic study of the interlinked factors of healthy diet and social support. 
Conclusion

In sum, this qualitative pilot study was conducted to identify sociocultural factors that may be contributing to Hispanic women having better birth outcomes than women of other ethnic groups in the epidemiological literature, referred to as the Hispanic Health Paradox. This trend also extends to Hispanic women who are recent immigrants to the U.S. having better birth outcomes than women who have resided in the U.S. for longer periods of time. Study participants primarily attributed the Hispanic Health Paradox to the consumption of a healthy diet consisting of home-cooked meals made of fresh, minimally processed ingredients. They identified social support as a secondary factor contributing to the paradox. Social support was ideally provided by the participants’ mother and included help with healthy meal preparation. These two prevalent responses—diet and social support—are actually one linked response. Many times a healthy diet was not possible without social support, and several times the social support featured cooking as a focal point. Many participants linked food, family, and home all togetherwith the love and support that that implies.

Even though the participants identified diet as an important factor that may explain differences in birth outcomes between Hispanic women who resided for different lengths of time in the U.S., they themselves did not differ in their reported consumption patterns while pregnant. This may be because the English speaking participants were highly educated, which is associated with better dietary habits among Hispanics who have lived in the U.S. for longer periods of time. Although many of the English-speaking participants worked outside the home while pregnant, they chose not to sacrifice the quality of their diet, but instead developed strategies for increasing the convenience of healthy food, including the activation of their social network. 
As a next step, a systematic biocultural study needs to be carried out to assess the extent of both the cultural construct of the ideal pregnancy dietary pattern among Hispanics and the components of its implementation, its relationship to social support, and the potential for such a pattern to be a protective factor in pregnancy and help explain the Hispanic Health Paradox. More qualitative, quantitative and mixed-methods studies about diverse types of protective factors, like home-cooked healthy diets and comprehensive social support, may be able to help a broad range of women by leading to guidance for interventions and evidence-based health practices. 
References Cited

Abu-Saad, Kathleen, and Drora Fraser. 2010. Maternal nutrition and birth outcomes. Epidemiologic Reviews 32 (1):5-25.

Almeida, J., B. E. Molnar, I. Kawachi, and S. V. Subramanian. 2009. Ethnicity and nativity status as determinants of perceived social support: testing the concept of familism. Soc Sci Med 68 (10):1852-8.

Arcia, E., M. Skinner, D. Bailey, and V. Correa. 2001. Models of acculturation and health behaviors among Latino immigrants to the US. Soc Sci Med 53 (1):41-53.

Arias, E. . 2010. United States life tables by Hispanic origin. Washington, D.C.: National Center for Health Statistics.

Ayala, Guadalupe X., Barbara Baquero, and Sylvia Klinger. 2008. A systematic review of the relationship between acculturation and diet among Latinos in the United States: Implications for future research. Journal of the American Dietetic Association 108 (8):1330-1344.

Bank, World. 2017. Mortality rate, infant (per 1,000 live births). The World Bank [cited August 24, 2017 2017]. Available from http://data.worldbank.org/indicator/SP.DYN.IMRT.IN.

Behnke, Marylou, Vincent C. Smith, Committeeonsubstanceabuse, and Committeeonfetusandnewborn. 2013. Prenatal substance abuse: Short- and long-term effects on the exposed fetus. Pediatrics 131 (3):e1009-e1024.

Berkman, L. F. 2000. Social support, social networks, social cohesion and health. Soc Work Health Care 31 (2):3-14.

Berry, John W. 1997. Immigration, Acculturation, and Adaptation. Applied Psychology: An International Review 46 (1):29.

Bloomfield, Frank H. 2011. How is maternal nutrition related to preterm birth? Annual Review of Nutrition 31 (1):235-261.

Bruce, Giles. 2017. To save babies, homing in on a ZIP code in Fort Wayne. The Times of Northwest Indiana, February 1, 2017.

CDC. 2017. Infant mortality. Centers for Disease Control (CDC) [cited August 24, 2017 2017]. Available from https://www.cdc.gov/features/infantmortality/index.html.

Repeated Author. 2008. Community Health and Program Services (CHAPS): Health disparities among racial/ethnic populations. Atlanta: U.S. Department of Health and Human Services.

Ceballos, Miguel. 2011. Simulating the effects of acculturation and return migration on the maternal and infant health of Mexican immigrants in the United States: A research note. Demography 48 (2):425-436.

Chávez, Noel, Lisa Sha, Victoria Persky, Patricia Langenberg, and Erlinda Pestano-Binghay. 1994. Effect of length of US residence on food group intake in Mexican and Puerto Rican women. Journal of Nutrition Education 26 (2):79-86.

Cobas, José A. , Hector Balcazar, Mary B. Benin, Verna M. Keith, and Yinong Chong. 1996. Acculturation and low-birthweight infants among Latino women: A reanalysis of HHANES data with Structural Equation Models. American Journal of Public Health 86 (3):394-396.

Cohen, G. R., L. B. Curet, R. J. Levine, M. G. Ewell, C. D. Morris, P. M. Catalano, D. Clokey, and M. A. Klebanoff. 2001. Ethnicity, nutrition, and birth outcomes in nulliparous qomen. American Journal of Obstetrics and Gynecology 185 (3):660-667. 
Cohen, S., and T. A. Wills. 1985. Stress, social support, and the buffering hypothesis. Psychol Bull 98 (2):310-57.

Collins, N. L., C. Dunkel-Schetter, M. Lobel, and S. C. Scrimshaw. 1993. Social support in pregnancy: psychosocial correlates of birth outcomes and postpartum depression. J Pers Soc Psychol 65 (6):1243-58.

Deyo, R. A., A. K. Diehl, H. Hazuda, and M. P. Stern. 1985. A simple language-based acculturation scale for Mexican Americans: validation and application to health care research. Am J Public Health 75 (1):51-5.

Dixon, Lori Beth, Jan Sundquist, and Marilyn Winkleby. 2000. Differences in energy, nutrient, and food intakes in a US sample of Mexican-American women and men: Findings from the Third National Health and Nutrition Examination Survey, 1988-1994. American Journal of Epidemiology 152 (6):548-557.

Dominguez, Kenneth, Ana Penman-Aguilar, Man-Huei Chang, Ramal Moonesinghe, Ted Castellanos, Alfonso Rodriguez-Lainz, and Richard Schieber. 2015. Vital signs: Leading causes of death, prevalence of diseases and risk factors, and use of health services among Hispanics in the United States - 2009-2013. Morbidity and Mortality Weekly Report (MMWR) 64 (17):469-478.

Feldman, P. J., C. Dunkel-Schetter, C. A. Sandman, and P. D. Wadhwa. 2000. Maternal social support predicts birth weight and fetal growth in human pregnancy. Psychosom Med 62 (5):715-25.

Fitzgerald, Nurgül. 2010. Acculturation, socioeconomic status, and health among Hispanics. NAPA Bulletin 34 (1):28-46.

Fleuriet, K. Jill. 2009. Problems in the Latina paradox: measuring social support for pregnant immigrant women from Mexico. Anthropology \& Medicine 16 (1):49-59.

Franzini, L. , J.C. Ribble, and A.M. Keddie. 2002. Understanding the Hispanic paradox. In Race, Ethnicity, and Health: A Public Health Reader, edited by T. A. LaVeist. San Francisco: Jossey-Bass.

Franzini, Luisa, John C Ribble, and Arlene M Keddie. 2001. Understanding the Hispanic Paradox. Ethnicity \& Disease 11 (3):496.

Friese, Susanne. 2014. Qualitative data analysis with ATLAS.ti. 2nd ed. Thousand Oaks, CA: Sage.

Gray, Virginia B, Jeralynn S Cossman, Wanda L Dodson, and Sylvia H Byrd. 2005. Dietary acculturation of Hispanic immigrants in Mississippi. Salud Pública de México 47 (5):351-360.

Gress-Smith, J. L., D. S. Roubinov, R. Tanaka, K. Cmic, N. Gonzales, C. Enders, and L. J. Luecken. 2013. Prenatal expectations in Mexican American women: development of a culturally sensitive measure. Arch Womens Ment Health 16 (4):303-14.

Gutierrez, Yolanda M. 1999. Cultural factors affecting diet and pregnancy outcome of Mexican American adolescents. Journal of Adolescent Health 25 (3):227-237.

Harley, K., and B. Eskenazi. 2006. Time in the United States, social support and health behaviors during pregnancy among women of Mexican descent. Soc Sci Med 62 (12):3048-61.

Harley, Kim, and Brenda Eskenazi. 2006. Social epidemiology: Time in the United States, social support and health behaviors during pregnancy among women of Mexican descent. Social Science \& Medicine 62:3048-3061. 
Harley, Kim, Brenda Eskenazi, and Gladys Block. 2005. The association of time in the US and diet during pregnancy in low-income women of Mexican descent. Paediatric and Perinatal Epidemiology 19 (2):125-134.

Himmelgreen, David, Nancy Romero Daza, Elizabeth Cooper, and Dinorah Martinez. 2007. “I don't make the soups anymore”: Pre-to post-migration dietary and lifestyle changes among Latinos living in West-central Florida. Ecology of Food and Nutrition 46 (56):427-444.

Hummer, RobertA, DanielA Powers, StarlingG Pullum, GingerL Gossman, and W. Parker Frisbie. 2007. Paradox found (again): Infant mortality among the Mexican-origin population in the United States. Demography 44 (3):441-457.

ISDH. 2010-2014 infant mortality rates by zip code. Epidemiology Resource Center: Indiana State Department of Health (ISDH).

Kieffer, Edith C., Diana B. Welmerink, Brandy R. Sinco, Kathleen B. Welch, Christina Y. Schumann, and Virginia Uhley. 2013. Periconception diet does not vary by duration of US residence for Mexican immigrant women. Journal of the Academy of Nutrition and Dietetics 113 (5):652-658.

Lara, M., C. Gamboa, M. I. Kahramanian, L. S. Morales, and D. E. Bautista. 2005. Acculturation and Latino health in the United States: A review of the literature and its sociopolitical context. Annual Review of Public Health 26:367-97.

LaVeist, Thomas A. 2005. Minority populations and health: An introduction to health disparities in the United States. Vol. 5: Jossey-Bass.

Lopez-Class, Maria, Felipe González Castro, and Amelie G. Ramirez. 2011. Conceptions of acculturation: A review and statement of critical issues. Social Science \& Medicine 72 (9):1555-1562.

Markides, K. S., and J. Coreil. 1986. The health of Hispanics in the Southwestern United States: An Epidemiologic Paradox. Public Health Reports 101 (3):253-65.

Mathews, TJ , and MF MacDorman. 2011. Infant mortality statistics from the 2007 period linked birth/infant death data set. National Vital Statistics Reports 59 (6):1-31.

Medicine, Institute of. 2006. Dietary Reference Intakes: The Essential Guide to Nutrient Requirements. Edited by J. J. Otten, J. P. Hellwig and L. D. Meyers. Washington, DC: The National Academies Press.

Mulvaney-Day, N. E., M. Alegria, and W. Sribney. 2007. Social cohesion, social support, and health among Latinos in the United States. Soc Sci Med 64 (2):477-95.

Palloni, A., and J. D. Morenoff. 2001. Interpreting the paradoxical in the Hispanic Paradox: Demographic and epidemiologic approaches. Annals of the New York Academy of Sciences 954:140-74.

Pérez-Escamilla, Rafael, and Predrag Putnik. 2007. The role of acculturation in nutrition, lifestyle, and incidence of Type 2 Diabetes among Latinos. The Journal of Nutrition 137 (4):860-870.

Rodriguez, N., C. B. Mira, N. D. Paez, and H. F. Myers. 2007. Exploring the complexities of familism and acculturation: central constructs for people of Mexican origin. Am J Community Psychol 39 (1-2):61-77.

Sarason, B. R., I. G. Sarason, D. Perlman, and A. L. Vangelisti. 2006. Close Relationships and Social Support: Implications for the Measurement of Social Support. In Cambridge Handbook of Personal Relationships. New York, NY: Cambridge University Press. 
Schaffer, Donna M., Ellen M. Velie, Gary M. Shaw, and Karen P. Todoroff. 1998. Energy and nutrient intakes and health practices of Latinas and White Non-Latinas in the 3 months before pregnancy. Journal of the American Dietetic Association 98 (8):876-884.

Shin, Y., and G. Maupome. 2017. Segmentation of Mexican-Heritage Immigrants: Acculturation Typology and Language Preference in Health Information Seeking. J Immigr Minor Health 19 (5):1163-1173.

Singh, G.K. , and S. M. Yu. 2002. Adverse pregnancy outcomes: Differences between U.S.- and foreign-born women in major U.S. racial ethnic groups. In Race, ethnicity, and health: A public health reader, edited by T. A. LaVeist. San Francisco: Jossey-Bass.

Thomson, Maria D., and Laurie Hoffman-Goetz. 2009. Defining and measuring acculturation: A systematic review of public health studies with Hispanic populations in the United States. Social Science \& Medicine 69 (7):983-991.

Unger, Jennifer B., Anamara Ritt-Olson, Karla Wagner, Daniel Soto, and Lourdes BaezcondeGarbanati. 2007. A Comparison of Acculturation Measures Among Hispanic/Latino Adolescents. Journal of Youth \& Adolescence 36 (4):555-565.

Viruell-Fuentes, E. A., J. D. Morenoff, D. R. Williams, and J.S. House. 2013. Contextualizing nativity status, Latino social ties, and ethnic enclaves: An examination of the 'Immigrant Social Ties Hypothesis’. Ethnicity \& Health 18 (6):586-609.

Viruell-Fuentes, Edna A., and Amy J. Schulz. 2009. Toward a dynamic conceptualization of social ties and context: Implications for understanding immigrant and Latino health. American Journal of Public Health 99 (12):2167-2175.

Wakeel, Fathima, Whitney P. Witt, Lauren E. Wisk, Michael C. Lu, and Shin M. Chao. 2014. Racial and ethnic disparities in personal capital during pregnancy: Findings from the 2007 Los Angeles Mommy and Baby (LAMB) Study. Maternal and Child Health Journal 18 (1):209-222. 